\title{
ANALYSIS OF MARKETING INFORMATION SYSTEMS AND CONCEPTION OF AN INTEGRATED MARKETING INFORMATION SYSTEM
}

\author{
Veselina Ivanova Nedeva \\ Technical College, Yambol, Trakia University, Stara Zagora, Bulgaria \\ Correspondence to: Veselina Nedeva, Trakia University, Stara Zadora, Technical college, \\ 8600 Yambol, Bulgaria, Gr.Ignatiev Str. 38, tel. 046 66-91-78, E-mail: vnedeva@yahoo.com
}

\begin{abstract}
The article presents the results of a research in the field of the marketing information technologies. The subjects of the research are the marketing information systems, mainly the information systems used in the marketing business activities of the studied companies. 25 marketing information systems were covered. Data were collected in the period 1999-2002. A study was conducted on the basis of criteria and indices preliminarily worked out as well. Analysis was made, results were obtained and a concept for the building of a marketing information system of an integrated kind was proposed.
\end{abstract}

Key words: Information Technology, Data Warehouse, OLAP, Data Mining, Integration, Database, Internet, Web.

\section{INTRODUCTION}

In the creation of the marketing information systems (MIS) the concrete needs of the organization, together with managing process of the organization's marketing activity are reported. Each company has its specific characteristics of an internal kind (production, price, distributing net, connections with other companies) and of an external kind (markets, competition, customers etc.) influencing the process of decision making. There is an objective need of information about the internal and the external factors and of a system for the information's managing necessary for carrying out of a marketing activity.
The following processes are studied in great detail the information assurance in the system, the information readiness for making decisions, the system brightness and the opportunities resulting from the use of new information and communication technologies.

\section{SUBJECT, CRITERIA AND METHODS OF RESEARCH}

The subjects of the research are the information systems in service of the marketing activity of companies also called marketing information systems (Table 1).

Table 1 - Analysed Marketing Information Systems

\begin{tabular}{r|ll}
\hline № & \multicolumn{1}{c}{ MIS name } & Company \\
\hline 1 & iLuxo Suite & iLux Co. \\
\hline 2 & 3M GEDW & Teradata Comp.,a devision of NCR \\
\hline 3 & Arena R & Systems Modeling Corporation \\
\hline 4 & Charles Schwab & Charles Schwab \& Co \\
\hline 5 & Clarify eFrontOfficeC & Amdocs Ltd. \\
\hline 6 & DIALOG++ & Balder Technology Group Inc. \\
\hline 7 & EDGE & AIT Group Plc. \\
\hline 8 & Enterprise Rent-A-Car's & CIO Service Inc. \\
\hline 9 & HTS & HNC Software Inc. \\
\hline 10 & Hummingbird & Hummingbird Ltd. \\
\hline
\end{tabular}




\begin{tabular}{|l|ll}
11 & iLuxo Suite & iLux Co. \\
\hline 12 & Imparto Suite & Primus Knowledge Solutions Inc. \\
\hline 13 & Inquisite & Catapult Systems Corp. \\
\hline 14 & IQSupport Application Suite & Logica Advantagekbs Inc. \\
\hline 15 & LeadMaster TM & Lead Master Inc. \\
\hline 16 & Owens \& Minor & Owens \& Minor Inc. \\
\hline 17 & JJM interconnection & PJM Interconnection L.L.C. \\
\hline 18 & Plan Magic & Plan Magic Corp. \\
\hline 19 & QAD & QAD Inc. \\
\hline 20 & Quadstone & Quadstone Company \\
\hline 21 & Quintus eContact TM Suite & Avaya inc. \\
\hline 22 & SalesLogix R & QGate Software Limited \\
\hline 23 & TeamPOINT & Point Corp. \\
\hline 24 & Tyson Foods & Optio Software Inc. \\
\hline 25 & VANTIVE & VANTIVE Inc. (PeopleSoft Inc.) \\
\hline & &
\end{tabular}

For the purpose of the research series with different criteria were created bearing in mind two different trends: functional purpose and information technology purpose.

The criteria (used in our research) constructing the basis for an evaluation of the marketing information systems about their functional purpose are the following:

1.Customer orientation of marketing information systems;

2.Maintaining the marketing mix elements;

3.Marketing research;

4.Data analysis;

5.Current reporting capabilities;

6.Possibilities for marketing control;

7.Using of information from former periods under review (inherited systems);

8.Forecasting.

We evaluate the second trend on the basis of information technological criteria referring to the application of information and communication technologies in marketing information systems. The criteria for the information technological purpose are as follows:

1.Quality of information for making decisions;

2.Platform independence;

3.Information base;

4.Working environment architecture;

5.Operation in Internet;

6.Analysis technologies;

7.Information protection.

The criteria of this group don't allow an unification or grouping and they are to an sufficient degree segmented in order the priorities or the shortcomings of the marketing information systems connected with the respective field of the information technologies to come to the fore, to evaluate their influence over the researched characteristics of the information and the information system. By some of the criteria evaluation indices are represented and their purpose is to analyse different techniques, technologies or methods for problem solving. If the marketing information system doesn't meet all indices after a given criterion it doesn't mean system incompleteness but it is connected with an other technological decision. Furthermore there are cases when no sufficient information for an evaluation of the marketing information systems after definite indices or criteria as a whole can be collected. Because of all these reasons and characteristics of the information technological criteria the evaluates subjectively and it reflects the researcher's view.

The criteria connected with the functional purpose as well the information technological criteria are complicated and to the scale:

* It complies with the index.

* It doesn't comply with the index.

* Without a valuation.

The observation and the content analysis are the main methods applied by researching. The observation specificity by this research is the studying of demo versions, products being placed at disposal for a definite limited time (usually a month) or using of products for a free realization of some tasks, registrations or investigations on the basis of researcher's data. The information obtained through observations was complemented and enriched by the content analysis. It was applied parallel to the observation. By determining the analysis categories, i.e. the most general key words one proceeds from the outlined criteria and indices. The indices selected meet the requirements for content analysis categories. They are comprehensive, mutually exclusive, reliable and appropriate. Some analysis 
units are applied, as the analysis is complicated: theme, contention and sometimes the entire text.

During the analysis process tables are used wherein the studied content analysis categories are registered.

\section{RESULTS}

The evaluation and the analysis of the marketing information systems using the functional criteria give a reason for making the following conclusion the following trends in the marketing activity of the companies are best developed and most completely included:

* Studying, giving prognosis and influence over the customer's behaviour - Imparto Suite, Quadstone, Claryfy eFront

Office, Quintus eContact TM Suite, DIALOG++, EDGE;

* Advertising -iLux Suite, Inquisite;

* Direct marketing - VANTIVE, Imparto Suite, Inquisite;

* Marketing planning - PlanMagic, IQSupport Application Suite;

* Sale managing - HTS, SalesLogix etc.

Customer orientation of MIS $(87 \%$ of MIS meet the requirements). Information about customers is of the greatest significance in information database. This information is used to study customers' behaviour, forecast their future behaviour or purchases, and influence on customers' behaviour with the purpose of keeping current customers and increasing their loyalty. On the grounds of it customers' satisfaction and dissatisfaction can be measured, and factors influencing their satisfaction and dissatisfaction are detected. MIS whose major tasks are executing marketing campaigns are focused on market segmentation, development, market campaigns implementation and studying their results.

Analysis of Market information systems by indicators assessing maintaining the marketing mix elements $(56 \%$ of MIS meet the requirements). shows that they are predominantly information systems whose database is built up by the data warehouse technology. In the rest it is difficult to enter information about customers and mix elements, and it is nearly impossible to integrate such information about the various objects. The systems maintaining such information are aimed at improving and maintaining products, selecting price strategy for maintaining competitive prices, comparative analysis of competitive products, advertising through the internet, direct marketing.

Possibilities for marketing research $(61 \%$ of MIS meet the requirements). . The conclusion is that through Web technologies coupled with the
Data warehouse and Data Mining, the processes for preparation, implementation and collecting information for marketing research may be automatized, and the information may be summarized and prepared for a subsequent analysis, as well.

Data analysis $(72 \%$ of MIS meet the requirements). . Analysing data from the MISs involves various aspects, but most frequently these are data about services (Arena R, Enterprise Rent-ACar's, PJM Interconnection) and sales (TeamPOINT, Charles Schwab, Hummingbird, 3M). Customers' behaviour is analysed in MIS which have a customer orientation. In analyzing MIS it was found that they maintain data mainly on marketing financial analysis, and namely - net profit, net sales, total assets etc.

Analysis of MIS by the criterion current reporting capabilities $\mathbf{( 7 0 \%}$ of MIS meet the requirements). shows that in $70 \%$ of MIS information is collected from well-prepared sources of current data. Disadvantages: they do not provide information on all marketing problems which marketing specialists generally solve. Opportunities for purposeful collection of information from suggestions and complaints from customers are not available and used in all MIS. They are particularly suitable for receiving feedback from customers, with the purpose of better satisfaction of their needs. (EDGE, Inquisite, LeadMasterTM, Enterprise RentA-Car's etc.)

Possibilities for marketing control $(54 \%$ of MIS meet the requirements). Information mainly on the chief maintained objects - products, customers, commercial channels, orders, is maintained for the profitability control; there is less data about market segments, territories. With regard to the yearly plan control only some indicators may be studied, depending on the degree of completeness and comprehensiveness of the information in the system database. Most frequently data information on sales of companies maintaining product information and analysis of customer satisfaction in companies maintaining data about services, is maintained. More complete control can be exercised in those MIS whose DBs include data about more objects and those which have better capabilities for information analysis.

Research of MIS shows that inherited systems (50\% of MIS meet the requirements). are a good information source through which forecasts and long-term plans are made for marketing activity control. Such an approach for utilizing information about past periods is used in those having or building up a data warehouse, which enables storage of such information after its transformation. MIS using information from inherited systems are about 
$51 \%$ of all analysed ones. Information from inherited systems, no matter from what bearers it is and for what systems it is adjusted, is important in studying trends. Specialists believe that $70 \%$ of information in big corporations is in a mainframe environment. The situation is the same also in MIS which accumulate information from inherited systems. A smaller part of it is located in remote data centres. In this case, if the information is not integrated in the MIS, in order to obtain it, a great deal of messages, data transportation and waiting are generated until the information necessary for the solution preparation is provided. Such procedures are time-consuming, means are significant and for this reason, its integration into the database of MIS is, on the one hand, a prerequisite for increasing the information readiness degree for making a decision, and on the other hand, it improves its basic features.

Forecasting takes only a small part of assessed MIS (41\%). It is difficult to assess how effective the various forecasting methods are. However, assessments show only if there are opportunities for making different forecasts on the grounds of the information stored in the $\mathrm{DB}$ or in the data warehouse.

Functional criteria enable determining the data comprehensiveness provided by the MIS for accomplishing marketing activities, whereas through information and technological criteria technologies for storage, analysis, protection etc are assessed.

Information quality for decision-making (66\% of MIS meet the requirements). . The study demonstrates MIS that simultaneously apply Data warehouse and OLAP (On-Line Analytical Processing) technologies have a better information quality and increasing its readiness for making and supporting decisions. Nearly $95 \%$ of the studied systems with such a technology comply with the indicators $(\mathrm{Qad} \AA$, Hummingbird, TeamPOINT, VANTIVE etc), whereas MIS with databases show relatively bad results (Arena ${ }^{\circledR}$, HTS, Imparto Suite, Inquisite, IQSupport Application Suite etc). There is no information system which completely satisfies some of the indicators. It is worth noting that MIS, which are assessed by this criterion, provide high quality information. The interactive reporting that they provide is based on changes in real time and develops in the following respects - opportunity for updating report data; opportunity for setting parameters for carrying out the research; opportunity for planning, distribution and sending results in different forms.

Information base $(50 \%$ of MIS meet the requirements). MIS have an information base built up through a relational Database or Data Warehouse (DW) technology. The correlation of information systems is to the favour of the Database but DW has an advantage with regard to degree of information generalization. The research confirmed the existing practice and theoretical abilities of the Data base for building one or a few tasks for marketing activities control: implementing marketing campaigns (iLuxo Suite, Imparto Suite, Quintus eContact ${ }^{\mathrm{TM}}$ Suite etc.); marketing planning (PlanMagic, IQSupport Application Suite); management of sales and direct marketing (HTS); remote, telephone services and sales management (SalesLogix $\left.{ }^{\circledR}\right)$ etc.

Platform independence. Performance of MIS on different computer platform and operation environments creates convenience and adaptability when the system hardware and software are changed. In order to maintain the date bases in the network environment this is almost a must since the two-way data transfer, from and to the MIS, slows down. The results from the analysis of MIS show the following: opportunities for operation in the most common environments (Windows, IBM OS/2, Apple Macintosh, UNIX) - 1/5 от МИС (VANTIVE, Inquisite, Imparto Suite и др.); data transferability from and to architectural environments different from that of the MIS approximately $70 \%$ of MIS (Enterprise Rent-ACar's, Qad $\AA$, Tyson Foods etc.); there is no information about a part of MIS by the one and/ or the other indicator (IQSupport Application Suite, Quintus eContact ${ }^{\mathrm{TM}}$ Suite, EDGE).

A large part of MIS are oriented towards Windows operation environment. The study shows that MIS should be capable of communicating data on IBM OS/2, Apple Macintosh, UNIX etc. This is necessary because by using SQL, network connection etc. the application can connect with the database located on a remote computer.

Architecture of the working environment. Two approaches are applied in MIS in creating the architecture of the working environment - 2-tier and multi-tier (N-tier) technology. Only about $1 / 3$ of MIS apply the first technology which does not provide the necessary speed of work and enough flexibility of structures for using web technologies. The other $2 / 3$ of them use the N-tier approach for building up the customer/server architecture. The architecture, through the $\mathrm{N}$-tier approach provides scalability of the applications, which may be easily, developed further through LAN, WAN, Internet and Intranet.

The research shows that the importance of this architecture influences time for accomplishing tasks of the MIS in the following way - it minimizes the net traffic, uses a separate server for statistical analyses, modeling and backend analyses, enables working on a few orders simultaneously, provides a user control over the metadata, a better platform maintenance of the reference systems, a better 
centralization of the environment management, data transfer from orders and reports to the reference computers, carries out tasks for creating correspondence and control groups and algorithms for expanding and restricting of segments.

Operations in Internet $\mathbf{7 4 \%}$ of MIS meet the requirements). Quick interactive access to information in real time is a distinctive characteristic of the analysed MIS. They provide access to customers, competitors etc through web technologies. In many MIS web technologies are a way to access the external databases provided by specialized geodemographic information systems.

Research shows that web technologies bring about improvement of information quality in a few respects - scalability, continuity and management.

Analysis technologies $(60 \%$ of MIS meet the requirements). The study of the MIS by the criterion Analysis technologies enables us to conclude that the three basic technologies are the traditional statistical analysis techniques, OLAP and DM.

It was found that in MIS OLAP technology, i.e. the operating analytical processing in actual time is a means for data analysis as it is in the traditional correlation model, as well as in Data warehouse. Through it analysts and managers have the opportunity to get an insight into the data, by using a fast uniform operational access to a great deal of any kinds of information representation, obtained from the initial data, with the purpose of showing different aspects of the actual activity of the enterprise. The daily company operations are processed in some OLTPs (Online Transactional Processing). OLAP requires statistical, full data about long periods of time in which errors have been deleted. OLAP, however, processes only one transaction at a time per loading cycle, when the information loads in a batch in the system but can process thousands of orders per day simultaneously.

Problems solved in MIS by utilizing Data warehouse/OLAP:

With regard to customers - profitability analysis, analysis of sales to each customer and for each period, analysis of the company position in terms of sales, expenses analysis, analysis of the quality of services etc.

With respect to products - analysis of quality, of value, of deliveries, of the value depending on the changes in the production mix etc.

Other problems - analysis of the external marketing information in terms of prices, demographic conditions etc.

MIS using Data Mining have an advantage compared to those using the traditional analysis techniques, because of the fact that the data prepared through them, is of better quality for taking decisions. It is a result of a comprehensive study of the vast amount of available information on an observed object or phenomenon. This feature is even more evident when the information base is built up through the Data Warehouse technology since the research is carried out on thorough information with millions of records which describe the research object. Knowledge, included in the knowledge base is a model of a functional dependence between the variables of the studied multitude (purchasing behaviour models of customers, models for finding dependences etc.). In Data Mining dependencies existing in the data base are more obvious than in any other technology.

Information protection. MIS maintain information which is valuable, important, costly and confidential. For this reason protection and access restriction have a great importance for its existing. Three methods of protection from external destructive activities or forbidden access are used in them - device methods; software methods; cryptographic methods.

Information on the protection of MIS is not available for all of them. It is known that only two of them use device protection methods (Imparto Suite и Quintus eContact ${ }^{\mathrm{TM}}$ Suite). Combined usage of device and software protection methods is used too. They are built up in the points of connecting with Internet, restricting access from and to the network.

In the studied MIS a great importance is attached to data protection in the intranet environment, i.e. restricting employees' access to particular information. About 95\% of MIS have such a protection. It is usually organized in three levels users` level; group level; company level.

\section{CONCEPTION OF AN INTEGRATION MARKETING INFORMATION SYSTEM}

The complexity and the multifunctional character of the marketing activity as well as the many possibilities of the modern information technologies allow the marketing information technologies to be developed in the integration direction.

The conception about building integrated marketing information systems (IMIS) is based on the conclusions drawn during the research of the modern marketing information systems 1 and the application of the information technologies there in 2.

The integration is comparatively a new development trend in MIS. From an information technological point of view the integration can be considered in three main directions:

* Integration of data;

* Integration of information technologies; 
* Integration in Web and Internet.

In the first place lays the question about the integration of data as a main system resource. The main aspects in this research trend are two: integration of data sources and integration of data schemes.

The integration of data sources arises as result of the data diversity and the difficulty all potential information sources need for solving the system tasks to be foreseen. The analysis shows that the following main groups of data sources can be determined: inherited information systems, operative information systems and external sources.

The essence of the proposed concept for integration is based on the fact that an united warehouse is established ensuring its co-ordination and an aggregation of data being kept. The priority of the Data Warehouse to be used in MIS is based in the fact that the data in the warehouse are renovated, constantly accumulating new data conformable to the MIS tasks. This is an important aspect for the existence of MIS. Using Data Warehouse in the information systems the usual business terms are applied.

The integration of data schemes is the other aspect of the whole data integration. The coordination of data from different sources stems from the features of the data elements and from the metadata features.

The process of integration of data coming in from different sources includes several consecutive steps - extraction and transformation of elements, co-ordination and integration, filtration and aggregation of data elements3.

Integration of information technologies. Every operative and analytical IS can use different information technological solutions. Data Warehouse (DW) and OLAP (On-line Analytical Processing) are applied for the building of IMIS in the concept of the present study.

Both concepts presented don't compete but they mutually complement. They are directly mutually connected and integrated in IMIS. DW can be used as a source of aggregated and detailed data for analysis and the OLAP technology is carrying out the analysis of the integrated data.

Integration of IMIS in Web and Internet. The use of the Internet technologies is a new important feature of IMIS. The development of Internet technologies allows a grave enlargement of the integration concept. The special features and the functions of IMIS are very well corresponding with the possibilities proposed by the global Internet. In this sense the global Internet can be considered as: a resource for establishing of contacts with end users and the distant sections and employees of the company;
* Channel for producing of integrated direct marketing;

* Resource for advertising

* Information source for planning a marketing campaign

* Source of unplanned information from customers and company employees.

The current concept for MIS structure of an integrated kind can be generalized presenting it in two groups of components: nucleus (information base, IB) and modules. The information base can include Data Warehouse), (DW), On Line Analytical Processing (OLAP) and Data Mining (DM). The operations of IMIS about the users' service and the data preparation for their input in the DW involve construction of following modules:

* Module for user's service

* Module for information from operative systems

* Module for geodemographic information

* Module for information from inherited systems

* Module for unplanned information

* Module for virtual communication

Usually the module realizes a number of mutually connected functions jointly ensuring functionality inherent in the information system.

The Data Warehouse, the Analytical System (OLAP) and the System for Data Mining take a central place in the IMIS structure. They present the basis on which the functionality of IMIS is to be constructed and that's why we call them Information Base or Nucleus of IMIS. It is clear that the IMIS nucleus informatically ensures the system. Integration in several directions is realized in the IS nucleus: integration of data and integration of technologies. The data integration is realized by the Data Warehouse technology and at the same time the Module for virtual communication integrates the technology for data saving namely the Data Warehouse and the analysis technologies namely OLAP and Data Mining are integrated, IMIS as a whole in Internet.

\section{CONCLUSIONS}

The conclusions that were drawn are the following:

The Data Warehouse technology for construction of the information base most highly influences the integration of the subjects, the activities and the information sources in comparison with the rest of the technologies being examined. Its contribution to the information readiness for making decisions results from the possibility for detailed data and data of a different generalized level to be saved. It is a precondition for making a thorough OLAP and Data Mining because it places at a base for researching of the whole data multitude. 
The works in Internet and the Web technologies have a great influence over the information readiness for making decisions. The access to external data bases offered by the specialised geodemographic information systems presents an information source which can not be ignored because it enriches the marketing information systems providing data about changes of user's preferences, tastes, behaviour etc.

Data Mining technology offers techniques, which use technologies like artificial intellect for in depth research of great data volumes. Its application is most effective when it is combined with the Data Warehouse technology. The application of the technology for an Data Mining has an influence over the integration of the activities because it allows a combination between accountancies and data analysis for the generation of decisions.

The conducted research of the marketing information systems using functional criteria shows that complete information integration can be achieved satisfying the managing needs if all possible ways for its use are studied. The satisfying of the needs will be more effective if maximum information quantity and data knowledge, saved in the information base, is unloaded. The service of the specialists and the managing authorities will become more effective and quick if a part of the report materials are preliminarily prepared and saved in a ready form in the information base, if there is a possibility of their repeated use and an access to the information base by all persons from Internet.

The platform independence proves to be obligatory in order to improve the adaptability of the information system using the possibility that data from different platforms and operation mediums are transferred. The improvement of the quality of the information in the information system is to a significant degree influenced by the working environment architecture giving better results in regard to the service speed if the approach is orientated to the use of a multilayer $\mathrm{N}$-tier architecture. The security of the information is extremely important especially when there is an access to Internet and a big number of information system users.

The information readiness for support and making decisions is influenced by all information technologies enriching the information qualities, the visualization forms and the access ways to the information base. The information features depend on the possibility for bigger task number to be included being basic and obligatory in the research, report, analysis and control marketing activities.

The information base of the marketing information system should support detailed as well as generalized information. Prepared report documents, which have the ability to be disseminated in Internet and Intranet, should be saved together with the data. They should allow real-time access.

\section{REFERENCES}

1. Codd, E.F., S.B. Codd,C.T. Salley. Providing OLAP to User-Analysts: An IT Mandate, , E.F.Codd \& Associates, 1993

2. DW Data Preparation, Conference Presentation, SYM8DW Data 1098J Hill, GartnerGroup, 1998

3. ESOMAR, EMAC, AFM, Symposium on Information Based Decision Making in Marketing, Paris, November 1993

4. Hall, Curt, Data Warehousing for Business Intelligence, <www.dw-institute.com>, March 1999

5. Harrmon, P. "The Corporate Use of Object Technology", ObjectWorld, San Francisko, 1997

6. Nedeva,V.I., Application of information technologies in the marketing, UE, Varna, magazine "Izvestia", ISSN 1310-0343, No.4/1998

7. Nedeva,V.I., Analysis of modern marketing information systems, International Anniversary Scientific Conference "Economics and Society Development", Varna, 2000, vol.III, ISBN 924-210125-2 (Vol.3)

8. Peneva P.H., Veselina Iv.Nedeva, Fundamental problems of data converting in Data Warehouse, Scientific Session "Technique, technologies and education", Yambol, 2001

9. Nedeva, V. I., Internet technologies in the modern marketing information systems, Scientific works, Vol.XLIV, Issue 3, Higher Institute of Food and Flavour Industries, Plovdiv, 2000

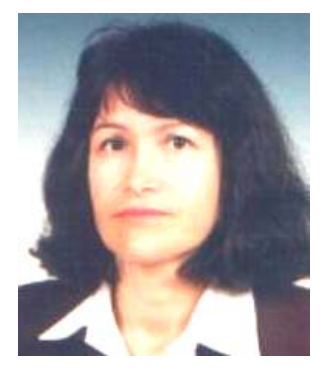

Veselina Ivanova Nedeva was born on January $18^{\text {th }}$, 1955 in the town of Yambol. She graduated from Economical high school "Georgi S. Rakovski" - Yambol in 1974. She got her university degree in "Informatics of economics" from the

Economical University -Varna in 1979.

She worked as a programmer in the department for "Automatical systems for control" in "Regional production and communal service company"Yambol.

She works in Yambol College for practice education (nowadays Technical College - Yambol) since 1988. She is a lecturer in Informatics in the above-mentioned college since 1989.

In May, 2003 she became a doctor within the field of "Applications of computer techniques in Economics". 